\title{
Neoclassical Transport in Enhanced Confinement Toroidal Plasmas
}

\author{
Z. Lin, W. M. Tang, and W. W. Lee \\ Princeton Plasma Physics Laboratory, Princeton University, Princeton, NJ 08543
}

It has recently been reported that ion thermal transport levels in enhanced confinement tokamak plasmas have been observed to fall below the "irreducible minimum level" predicted by standard neoclassical theory. This apparent contradiction is resolved in the present analysis by relaxing the basic neoclassical assumption that the ions orbital excursions are much smaller than the local toroidal minor radius and the equilibrium scale lengths of the system.

PACS: 52.25.Fi, 52.55.-8, 52.65.-y

The striking improvement of plasma confinement to neoclassical levels was first theoretically predicted for a reversed magnetic shear configuration [1] and subsequently confirmed in recent tokamak experiments [2-4]. In the so-called enhanced reversed shear (ERS) regime [2], the core ion thermal conductivity was actually observed to fall below the standard neoclassical level which was widely accepted as the irreducible minimum. The source of this apparent contradiction lies in the fact that the experimental conditions in the ERS regime correspond to situations where the ion poloidal gyroradius $\rho_{p}$ can be comparable in magnitude or even greater than the equilibrium pressure gradient scale length $L_{p}$ and/or the local minor radius $r$. This violates a basic assumption in the standard neoclassical formalism and establishes the need for a revised theory where $\rho_{p}$ can be of the same order of magnitude as $L_{p}$ and/or $r$. This is accomplished in the present analysis, and the associated analytical and gyrokinetic particle simulation results are shown to be in agreement with key confinement trends observed in ERS plasmas.

The standard neoclassical theory assumption that the ion poloidal gyroradius is much smaller than the local minor radius and the equilibrium scale lengths allows the expansion of the equilibrium distribution function around a local Maxwellian which is defined as a function of the flux surface. However, due to the combination of high central $q$ (safety factor) and small local inverse aspect ratio ( $r / R_{0}$ with $R_{0}$ being the tokamak major radius), the ion poloidal gyroradius can in fact be larger than the minor radius and comparable to the pressure gradient scale length in the ERS regime. Hence, both the trapped particle fraction and the banana width estimates can be significantly modified.

In the present analysis, the newly derived form for the neoclassical ion heat conductivity is found to be strongly reduced by finite banana width dynamics in the ERS regime. Important properties which need to be taken into account in an appropriate theoretical model include: (a) ion banana width is nearly constant close to the magnetic axis and (b) counter-moving ions have a minimum trapped fraction and all co-moving ions are trapped. In the usual neoclassical picture, outward ion heat conductivity results from energy flux imbalance between the inward moving slow (lower energy) particles and the outward moving fast (higher energy) particles. When the finite orbit width is taken into account, the outward ion heat conductivity is significantly reduced because this modification is much stronger on the fast particles (i.e., net energy outflow reduced). Therefore, the ion heat conductivity $\chi_{i}$ decreases for smaller minor radius where orbit effects are strongest. In the following, it will be demonstrated that analytic results from a random-walk type argument and global gyrokinetic particle simulations using the GNC code [5] yield favorable agreement with key trends from the experimental measurements.

In an axisymmetric system, the guiding center trajectories are defined by the conservation of magnetic moment, $\mu$, energy, $\varepsilon$, and toroidal canonical angular momentum, $p$, i.e.,

$$
\left\{\begin{array}{l}
\mu B+\frac{1}{2} m v_{\|}^{2}+e \Phi=\varepsilon \\
R\left(m v_{\zeta}+e A_{\zeta}\right)=p
\end{array}\right.
$$

where $B$ is the magnetic field, $m$ is the particle mass, $e$ is the charge, $v_{\zeta}$ is the toroidal component of the parallel velocity $v_{\|}, \Phi$ is the electrostatic potential, $R$ is the distance from the geometric center, and $\zeta$ denotes toroidal angle in the plasma current direction. The toroidal magnetic vector potential $A_{\zeta}$ is related to the poloidal magnetic field $\mathbf{B}_{\theta}=\nabla \times \mathbf{A}_{\zeta}$.

In analyzing the dynamics of interest, it is convenient to consider a high aspect ratio torus with concentric flux surfaces and a constant $q$ profile with constant $\Phi$. The focus here is on the orbit topology of single energy $\left(m v^{2} / 2\right)$ particles at a reference point of minor radius $r$ and poloidal angle $\theta=0$ (magnetic field minimum). The usual definition of a trapped particle is one for which $v_{\|}=0$ somewhere along the orbit. The banana width $\Delta_{b}$ and trapped fraction $f_{t}$ are determined by the behavior of barely trapped particles with $v_{\|}=0$ at the inside midplane $(\theta=\pi)$. In the limit of $\triangle_{b} \ll r$, standard analysis of Eq.(1) yields,

$$
\left\{\begin{array}{l}
f_{t}=\sqrt{2 \epsilon} \\
\triangle_{b}=\sqrt{8} \rho q / \epsilon^{1 / 2}
\end{array}\right.
$$

where $\epsilon=r / R_{0}$ and $\rho=v / \Omega$ with $\Omega \equiv e B / m$.

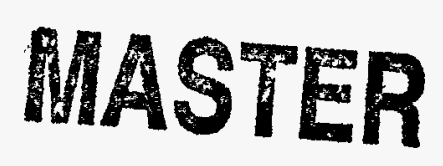




\section{DISCLAIMER}

This report was prepared as an account of work sponsored by an agency of the United States Government. Neither the United States Government nor any agency thereof, nor any of their employees, make any warranty, express or implied, or assumes any legal liability or responsibility for the accuracy, completeness, or usefulness of any information, apparatus, product, or process disclosed, or represents that its use would not infringe privately owned rights. Reference herein to any specific commercial product, process, or service by trade name, trademark, manufacturer, or otherwise does not necessarily constitute or imply its endorsement, recommendation, or favoring by the United States Government or any agency thereof. The views and opinions of authors expressed herein do not necessarily state or reflect those of the United States Government or any agency thereof. 


\section{DISCLAIMER}

Portions of this document may be illegible in electronic image products. Images are produced from the best available original document. 
Now consider the critical region, $\Delta_{b} \sim r$, where the familiar expressions given by Eq. (2) are invalid. First, note that the banana width increases when the reference point moves toward the magnetic axis. Hence, care must be taken to satisfy the basic constraint that the orbit excursion cannot be larger than the local minor radius at the outermost point of the orbit. For a small value of $r$, it is possible for the guiding center to pass through the magnetic axis at a local minor radius. Then, the banana width is equal to this local minor radius, i.e., it becomes the maximum banana width of the system. Secondly, the trapped orbit in this region is now re-defined as one in which a particle cannot travel around the poloidal angle. Because the guiding center drift does not cancel at each half of the orbit, this orbit can have a large radial excursion. Thus, it makes a large contribution to diffusion even though its parallel velocity $v_{\|}$may not change sign along the orbit. Near the magnetic axis, all co-moving particles $\left(v_{\|}>0\right)$ are trapped because the guiding center drift velocity is larger than the poloidal component of the parallel velocity. The counter-moving particle $\left(v_{\|}<0\right)$ has a minimum velocity space pitch angle at the trappedpassing boundary.

In order to quantify the deviation from the usual neoclassical transport estimates, we begin by considering barely trapped counter-moving particles which are initially located at the magnetic axis with parallel velocity $v_{\| 0}<0$. Such particles have zero parallel velocity at the inside mid-plane $(\theta=\pi)$ and later intercept the outer mid-plane $(\theta=0)$ at minor radius $r=\Delta_{\max }$ with parallel velocity $v_{\|}>0$. The maximum banana width $\Delta_{\max }$ and minimum trapped fraction $f_{\min }$ can be calculated from Eq. (1),

$$
\begin{aligned}
& \Delta_{\max }=\frac{(1+\sqrt{5})}{2^{1 / 3}}\left(q^{2} \rho^{2} R_{0}\right)^{1 / 3}, \\
& f_{\min } \equiv \frac{\left|v_{\| 0}\right|}{v}=\left(\frac{2 q \rho}{R_{0}}\right)^{1 / 3} .
\end{aligned}
$$

It is then clear that at minor radius $\Delta_{\max }$, the orbit topology begins to deviate from the usual neoclassical picture. For $r<\Delta_{\max }$, the banana width and trapped fraction are roughly constant and independent of local $\epsilon$. Furthermore, the ion collisionality parameter, $\nu_{i}^{*} \equiv \epsilon^{-3 / 2} \sqrt{2} q R_{0} /\left(v_{t h} \tau_{i}\right)$ with $v_{t h} \equiv \sqrt{2 T / m}$ being the thermal velocity and $\tau_{i}$ the ion Braginskii time, is finite and falls well within the banana regime for the ERS plasmas provided the actual trapped fraction is properly taken into account.

The usual estimates for the diffusion of passing (untrapped) particle guiding center orbits (Pfirsch-Schluter transport) and for gyroradius diffusion (classical transport) remain valid in the core ERS regime. However, the diffusion of trapped particles (banana transport) is expected to be dramatically changed due to the finite orbit width dynamics. In order to properly analyze such effects, the standard neoclassical formalism has been revised by relaxing the constraint that the ion orbital excursions must be smaller than the local minor radius and the equilibrium scale lengths of plasma. Results from gyrokinetic particle simulations based on this new formalism are strikingly different from the usual banana regime estimate and in reasonable agreement with experimental trends observed in ERS plasmas. Before displaying these numerical results, it is appropriate to first present useful analytical estimate for these important large orbit modifications. Significant physical insight can be gained from estimates of the ion energy flux $Q$ in the banana regime using the standard random walk approximation, i.e.,

$$
Q=\alpha \int_{0}^{\infty} f_{t} \frac{\Delta^{2}}{\tau_{\text {eff }}} \frac{\partial f}{\partial r} \frac{m v^{2}}{2} 4 \pi v^{2} d v,
$$

where $f$ is the ion guiding center distribution function, $\Delta$ is the random walk step size and $\tau_{e f f}=f_{t}^{2} / \nu$ is the effective collision time with $\nu$ being the ion collision frequency, and $\alpha$ is a simple constant which normalizes $Q$ to the standard neoclassical banana transport value calculated in the limit of small banana width. Since the temperature gradient scale length $\left(L_{T}\right)$ in ERS plasmas is much larger than the actual ion orbit width, local Maxwellian distribution can be assumed. Then the ion thermal conductive transport from Eq. (5) becomes

$$
Q=\alpha \frac{\kappa_{t} n T}{\tau_{i}} \int_{0}^{\infty} \frac{\triangle^{2}}{f_{t}} e^{-x}\left(x-\frac{3}{2}\right) d x
$$

with normalized energy $x=\left(v / v_{t h}\right)^{2}$. This results was obtained with a Lorentz collision model of the form, $C=$ $\nu(v) \hat{\mathbf{L}}$, where $\hat{\mathbf{L}}$ is the pitch angle scattering operator, and $\nu(v)=3 \sqrt{2 \pi} /\left(4 \tau_{i}\right)\left(v_{t h} / v\right)^{3}$. It is useful here to introduce an expression for the minor radius transition point for particles with thermal energy; i.e., Eq. (3) gives

$$
r_{0} \equiv \triangle_{\max }\left(v=v_{t h}\right)=\frac{(1+\sqrt{5})}{2^{1 / 3}}\left(q^{2} \rho_{i}^{2} R_{0}\right)^{1 / 3},
$$

where $\rho_{i}=v_{t h} / \Omega$. In terms of typical TFTR ERS plasma parameters, $r_{0} \sim 20 \mathrm{~cm}$. The normalized particle energy now becomes $x=\left(\triangle_{\max } / r_{0}\right)^{3}$ with the normalized minor radius defined by

$$
x_{0} \equiv\left(\frac{r}{r_{0}}\right)^{3} .
$$

When $x \geq x_{0}$, the particles reach their maximum banana width, and the orbit topology becomes different from the usual neoclassical picture. Therefore, the velocity space integral in Eq. (6) can be separated into two parts. Low energy particles $\left(x<x_{0}\right)$ have the conventional step size, $\Delta=q \rho / \sqrt{r / R_{0}}$, and trapped fraction, $f_{t}=\sqrt{r / R_{0}}$, while high energy particles $\left(x>x_{0}\right)$ have constant step size, $\Delta=q \rho / \sqrt{\Delta_{\max } / R_{0}}$, and trapped fraction, $f_{t}=$ $\sqrt{\triangle_{\max } / R_{0}}$. Equation. (6) then yields the result 


$$
Q=\alpha \frac{\kappa_{t} n T}{\tau_{i}} \frac{\rho_{i}^{2} q^{2}}{2 \epsilon^{3 / 2}}\left[1-e^{-x_{0}}\left(x_{0}+1\right)\right] .
$$

Accordingly, our analytical estimate for the finite-orbitmodified neoclassical ion heat conductivity in the banana regime becomes:

$$
\chi_{i}^{b a}=\chi_{i}^{n e o}\left[1-e^{-x_{0}}\left(x_{0}+1\right)\right],
$$

where $\chi_{i}^{\text {neo }}=0.66 \epsilon^{-3 / 2} q^{2} \rho_{i}^{2} / \tau_{i}$ is the standard neoclassical ion heat conductivity $[6,7]$. The correction factor, $f \equiv 1-e^{-x_{0}}\left(x_{0}+1\right)$, is shown in the Fig. 1. It is also of interest to note here that negative magnetic shear has a favorable effect on the orbit topology. Specifically, if $q \sim r^{-n}$, then $\Delta_{\max } \sim \varepsilon^{1 /(2 n+3)}$, a relationship first pointed out by Stix [8]. The contribution from the high energy particles to the integral in Eq. (6) decreases when the dependence of the maximum banana width on energy is weaker. Therefore, the negative magnetic shear helps to reduce the neoclassical ion heat conductivity in the core region.

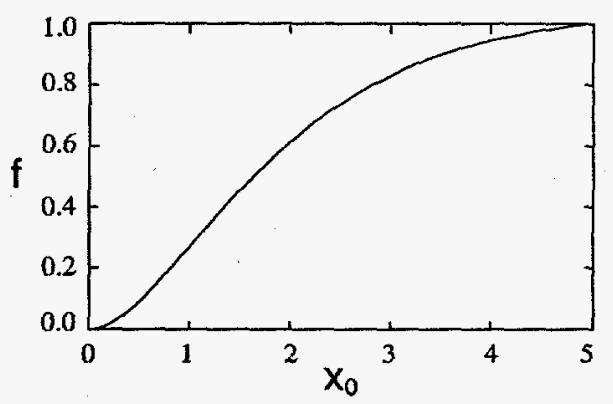

FIG. 1. Finite orbit width correction to standard neoclassical ion heat conductivity from Eq. (10).

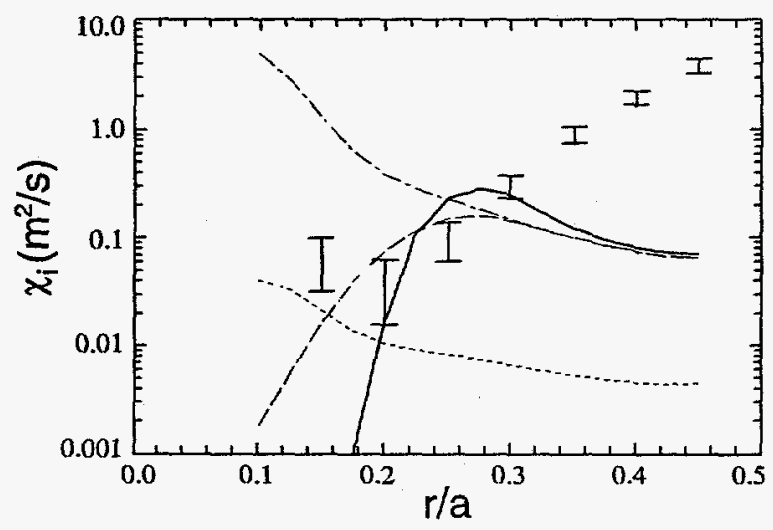

FIG. 2. Comparison of theoretical estimates of $\chi_{i}$ with experimental data. The solid line represents results of numerical solutions to the random walk model of Eq. (5), analytic results from Eq. (10) are represented by the dashed line, the dotted-dashed line represents the usual neoclassical results, and dotted line represents the Pfirsch-Schluter transport. Representative TFTR ERS experimental results are shown with error bars.

The total ion collisional heat conductivity is giving by

$$
\chi_{i}=\chi_{i}^{b a}+\chi_{i}^{p s}+\chi_{i}^{c l}
$$

with $\chi_{i}^{b a}$ being the focus of our present analysis, $\chi_{i}^{p s}=$ $1.58\left(1-f_{t}\right) q^{2} \rho_{i}^{2} / \tau_{i}$ representing the Pfirsch-Schluter transport [6], and $\chi_{i}^{c l}=\rho_{i}^{2} / \tau_{i}$ being the classical transport [9]. In the core region of ERS plasmas, the ion heat conductivity is close to the Pfirsch-Schluter level which is in turn much larger than the classical transport because of the high central $q$. Comparisons of the theoretical estimates for $\chi_{i}$ vs. representative TFTR ERS results are shown in Fig. 2. In contrast to the usual neoclassical values which strongly increase with decreasing minor radius, the finite orbit width analytic results from Eq. (10) and numerical results from Eq. (5) indicate a decreasing trend. This is in reasonable agreement with the earlier published TFTR ERS results [2] and even more strikingly with recent more comprehensive data analysis of the same representative discharge. Note also that the experimental trend begins to track the Pfirsch-Schluter level when $\chi_{i}^{b a}$ properly becomes negligibly small at smaller plasma radii.

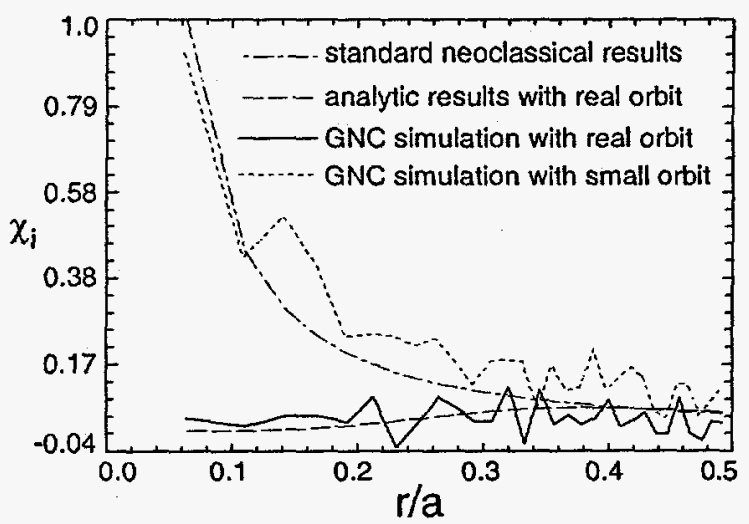

FIG. 3. Numerical and analytic estimates for $\chi_{i}$ in arbitrary units.

In order to validate the results from the random walk analysis, fully toroidal particle simulations using the gyrokinetic neoclassical code (GNC) [5] have been carried out. Since the temperature gradient scale length in ERS plasmas is much larger than the actual ion orbit width, the usual expansion around a local Maxwellian is justified and thus allows the use of a noise-reduction $\delta f$ scheme. The nonlinear gyrokinetic delta-f formalism $[10,11]$ allows a rigorous treatment of both finite ion orbit width dynamics and a Fokker-Planck collision operator conserving momentum and energy. In the simulations, the guiding center distribution function is calculated by integrating the exact marker particles trajectories. The energy flux is calculated by the standard procedure of multiplying each particle energy by its radial drift velocity. Thus both banana and Pfirsch-Schluter diffusion are measured. The calculated ion thermal transports are not sensitive to the initial loading of the particles or the initial conditions. The simulation results, which are presented in 
Fig. 3, support the trends from the random walk analysis shown in Fig. 2 and indicate that the ion heat conductivity decreases to roughly the Pfirsch-Schluter level in the ERS core regime. Moreover, if the key full ion orbit width dynamics are suppressed, the simulations recover the standard neoclassical results.

Despite the fact that estimates using the random walk models giving by Eq. (5) and Eq. (10) provide valuable physics insight and are well supported by the gyrokinetic particle simulations, it is nevertheless of interest to pursue an analytic derivation of neoclassical transport including finite orbit width dynamics. The equilibrium state with finite orbit width can be readily obtained using the principle of maximum entropy, and is found to be a global shifted-Maxwellian in an isolated system,

$$
f=\exp (a+b \varepsilon+c p)
$$

where $a, b$ and $c$ are numerical constants. This equilibrium state is characterized by uniform temperature and toroidal rotation, and no transport driven by ionion collisions. The characteristic density has the form, $n(\Psi)=\exp (\alpha \Phi+\beta \Psi)$, with $\Psi$ being the poloidal magnetic flux and $\alpha, \beta$ numerical constants. Because the density can be of arbitrary profile, the associated electrostatic potential can be of any form subject to the constraint of ion radial force balance. Thus, the equilibrium state derived here contains the orbit squeezing effect associated with the shear of the radial electric field [12]. For this constant temperature equilibrium, the poloidal flow can be shown to be zero, and the orbit squeezing effect accordingly does not change the neoclassical flows. The orbit squeezing effect on a general driven steady state (i.e. non-equilibrium state) is still a topic of ongoing debate $[13,14]$. Furthermore, when electron dynamics are considered in this model, it is found that the bootstrap current and ambipolar particle flux are consistent with the usual neoclassical results for any value of $\rho_{p} / L_{p}$.

In a general driven system, the steady state is the solution to a variational problem of minimum collisional entropy production subject to the constrains of external sources. Since it is not a zero entropy production state, transport can be driven by ion-ion collisions. In the case of constant temperature considered here, this means particle flux can be driven even though like-species collisions conserve momentum. Physically, this is a result of the conservation of toroidal angular momentum and its amplitude can be estimated accordingly ; i.e,

$$
\Gamma_{i} / \Gamma_{n e o} \sim \delta \frac{\mu_{i}}{\chi_{i}^{n e o}}\left(\frac{m_{i}}{m_{e}}\right)^{1 / 2} \frac{\rho_{p}}{r} \frac{\rho_{p}}{L_{n}}
$$

where $\mu_{i}$ is the neoclassical viscosity and $\Gamma_{n e o}$ is the usual neoclassical ambipolar flux. Here $\delta$ measures the degree of deviation from the equilibrium state and is defined as,

$$
\delta=-\frac{\kappa_{u}}{\kappa_{n}} \frac{u_{\zeta}}{u_{n e o}}
$$

where $u_{\zeta}$ is the toroidal mass flow, $\kappa_{2 \zeta}=-d \ln u_{\zeta} / d r$, $\kappa_{n}=-d \ln n / d r$, and $u_{n e o}=\rho_{p} \kappa_{n} v_{t h} / 2$. This scaling is similar to that obtained in very early classical transport studies [15] of particle flux arising from like-species collisions. Since $\delta$ is very small in most ERS plasmas of interest, it follows that steep density gradients do not have a strong effect on the ion heat conductivity. The orbit squeezing effects arising from the shear of the effective toroidal flow $\left(c E_{r} / B_{\theta}\right)$ driven by the radial electric field associated with the steep density gradient is small because the scale length of this flow is, although comparable to $\rho_{p}$, much larger than the actual ion banana width. These results further support the conclusion from the random walk calculations and the gyrokinetic simulation results.

The authors gratefully acknowledge S. Scott for providing key results from recent extensive transport analyses of TFTR data. We would also like to thank A. H. Boozer, T. S. Hahm, F. L. Hinton and M. C. Zarnstorff for critical reading of the manuscript, and M. A. Beer, R. V. Budny, F. M. Levinton and G. Rewoldt for valuable input on the interpretation of TFTR data. This work was supported by DoE Contract No. DE-AC02-76-CH03073, and in part by the U.S. DoE Fusion Energy Postdoctoral Research Program administered by the Oak Ridge Institute for Science and Education.

[1] C. Kessel, J. Manickam, G. Rewoldt, and W. M. Tang, Phys. Rev, Lett. 72, 1212 (1994).

[2] F. M. Levinton et al., Phys. Rev. Lett. 75, 4417 (1995).

[3] E. J. Strait et al., Phys. Rev. Lett. 75, 4421 (1995).

[4] T. Fujita et al., Phys. Rev. Lett. (to be published).

[5] Z. Lin, W. M. Tang, and W. W. Lee, Phys. Plasmas 2, 2975 (1995).

[6] F. L. Hinton and R. D. Hazeltine, Rev. Mod. Phys. 48, 239 (1976).

[7] R. D. Hirshman and D. J. Sigmar, Nucl. Fusion 21, 1079 (1981).

[8] T. H. Stix, Plasma Physics 14, 367 (1972).

[9] S. I. Braginskui, Reviews of Plasma Physics (Consultants Bureau, New York, 1965), Vol. 1.

[10] W. W. Lee, Phys. Fluids 26, 556 (1983).

[11] S. E. Parker and W. W. Lee, Phys. Fluids B 5, 77 (1993).

[12] R. D. Hazeltine, Phys. Fluids B 1, 2031 (1989).

[13] K. C. Shaing, C. T. Hsu, and R. D. Hazeltine, Phys. of Plasma 1, 3365 (1994).

[14] F. L. Hinton et al., Phys. Rev. Lett. 72, 1216 (1994).

[15] C. L. Longmire and M. N. Rosenbluth, Phys. Rev. 103, 507 (1956). 